PROCEEDINGS OF THE

AMERICAN MATHEMATICAL SOCIETY

Volume 138, Number 12, December 2010, Pages 4303-4309

S 0002-9939(2010)10426-4

Article electronically published on June 15, 2010

\title{
RECOVERING SINGULAR INTEGRALS FROM HAAR SHIFTS
}

\author{
ARMEN VAGHARSHAKYAN \\ (Communicated by Michael T. Lacey)
}

\begin{abstract}
We recover one-dimensional Calderón-Zygmund convolution operators with sufficiently smooth kernels by means of a properly chosen averaging of certain dyadic shift operators. As a corollary, a sharp $A_{2}$ inequality for these Calderón-Zygmund operators is derived from a corresponding inequality for dyadic shift operators.
\end{abstract}

\section{INTRODUCTION}

We will represent one-dimensional Calderón-Zygmund convolution operators with sufficiently smooth kernels (see conditions (2.5), (2.6) ) by means of a properly chosen averaging of certain Haar shift operators with bounded coefficients. By Haar shift operators we mean linear operators that can be expressed in an efficient manner with the Haar basis; see Remark 2.8.

The use of Haar shift operators to represent singular integral operators goes back to the work of T. Figiel 1. Later S. Petermichl derived a representation of the Hilbert transform [11. Similar representations were derived for Beurling [3], Riesz 13 transforms and the truncated Hilbert transform (S. Petermichl, oral communication). The reason why these representations are useful is that one can deduce deep facts about singular integral operators, based on the analysis of Haar shift operators. In Petermichl's original paper [11] a deep property of Hankel operators associated to matrix symbols was deduced. These representations also allowed to deduce the linear $A_{2}$ bound for the Hilbert [14] and Riesz transforms [12]. The study of the Haar shift operators is interesting in itself [8,9 and has become an important model of the singular integral operators; see for instance their use in [5, 6].

To illustrate this, as a corollary to our main result, Theorem 2.4 below, and the main result of [4, we see that we have a proved a sharp $A_{2}$ inequality for the Calderón-Zygmund operators, a question of current interest:

Corollary 1.1. Let

$$
T(f)(x)=P . V . \int_{\mathbb{R}} K(x-t) f(t) d t
$$

Received by the editors January 28, 2010.

2010 Mathematics Subject Classification. Primary 42B20, 42A45.

This research was supported in part by NSF grant 0456611 .

(C)2010 American Mathematical Society Reverts to public domain 28 years from publication 
be a one-dimensional Calderón-Zygmund convolution operator whose kernel $K$ is odd and satisfies (2.5) and (2.6). Then

$$
\|T f\|_{L_{2}(\omega)} \lesssim\|\omega\|_{A_{2}}\|f\|_{L_{2}(\omega)} .
$$

$B y\|\omega\|_{A_{2}}$ we mean the $A_{2}$ constant of the weight $\omega$. (See [7,14, for a definition.)

This generalizes the result of S. Petermichl, obtained for the Hilbert transform [14, and improves the estimates of A. Lerner, S. Ombrosi and C. Pérez [7, equation 1.9] for these particular types of Calderón-Zygmund operators.

\section{Formulation of the Result}

In order to formulate the main theorem, we introduce some notation.

For any $\beta=\left\{\beta_{l}\right\} \in\{0,1\}^{\mathbb{Z}}$ and for any $r \in[1,2)$ define the dyadic grid $\mathbb{D}_{r, \beta}$ to be the collection of intervals

$$
\mathbb{D}_{r, \beta}=\left\{r 2^{n}\left([0 ; 1)+k+\sum_{i<n} 2^{i-n} \beta_{i}\right)\right\}_{n \in \mathbb{Z}, k \in \mathbb{Z}} .
$$

This parametrization of dyadic grids appears explicitly in 2] and implicitly in [10, section 9.1]. Note that the dyadic grid we use is different from the one used in [11.

Place the usual uniform probability measure $\mathbb{P}$ on the space $\{0,1\}^{\mathbb{Z}}$; explicitly

$$
\mathbb{P}\left(\beta: \beta_{l}=0\right)=\mathbb{P}\left(\beta: \beta_{l}=1\right)=\frac{1}{2}, \quad \text { for all } l \in \mathbb{Z} .
$$

We define two functions. Take $h$ to be the function supported on $[0,1]$ defined by

$$
h(x)= \begin{cases}7, & 0<x<1 / 4 \\ -1, & 1 / 4 \leq x \leq 1 / 2 \\ 1, & 1 / 2 \leq x \leq 3 / 4 \\ -7, & 3 / 4 \leq x \leq 1\end{cases}
$$

and $g$ to be the function supported on $[0,1]$ defined by

$$
g(x)= \begin{cases}-1, & 0 \leq x \leq 1 / 4 \\ 1, & 1 / 4 \leq x \leq 1 / 2 \\ 1, & 1 / 2 \leq x<3 / 4 \\ -1, & 3 / 4 \leq x \leq 1\end{cases}
$$

Note that the function $g$ appears in [11] paired with the usual Haar function. In contrast, our function $h$, defined by (2.1), differs a little bit from the Haar function. In some sense, our choice of the function $h$ makes the convolution $h * g$ 'less smooth', and this property will be crucial for the proof. We will make this statement precise in (3.12), which will permit us to invert a Fourier transform.

For any function $f$ and any interval $I=[a, a+l]$ we define the function $f_{I}$ to be the scaling of $f$ to $I$ which preserves the $L_{2}$-norm; namely

$$
f_{I}(x)=f_{[a, a+l]}=\frac{1}{\sqrt{l}} f\left(\frac{x-a}{l}\right) .
$$

Now we are ready to state our main theorem: 
Theorem 2.4. Let $K:(-\infty, 0) \cup(0, \infty) \rightarrow \mathbb{R}$ be an odd, twice differentiable function (in the sense that $K^{\prime}$ is absolutely continuous) which satisfies

$$
\lim _{x \rightarrow \infty} K(x)=\lim _{x \rightarrow \infty} K^{\prime}(x)=0
$$

and

$$
x^{3} K^{\prime \prime}(x) \in L_{\infty}(\mathbb{R}) .
$$

Then there exists a coefficient function $\gamma:(0, \infty) \rightarrow \mathbb{R}$ satisfying

$$
\|\gamma\|_{\infty} \leq C\left\|x^{3} K^{\prime \prime \prime}(x)\right\|_{\infty}
$$

so that

$$
K(x-y)=\int_{\{0,1\}^{\mathbb{Z}}} \int_{1}^{2} \sum_{I \in \mathbb{D}_{r, \beta}} \gamma(|I|) h_{I}(x) g_{I}(y) \frac{d r}{r} d \mathbb{P}(\beta)
$$

for all $x \neq y$. Here $C$ is some absolute constant and the series on the right of (2.7) is a.e. absolutely convergent.

Remark 2.8. Note that for fixed $r, \beta$ and a function $\gamma \in L_{\infty}(R)$, the linear operator

$$
f \mapsto \sum_{I \in \mathbb{D}_{r, \beta}} \gamma(|I|)\left\langle g_{I}, f\right\rangle h_{I}(x)
$$

is an example of a Haar shift operator as defined in 4. Note that this operator, expressed as a matrix in the Haar basis, has a bounded diagonal, but is even better than that: one only needs to use Haar coefficients associated with dyadic intervals that intersect and have lengths that differ by at most a factor of 2 .

\section{Proof of the Theorem}

Step 1: Derivation of an integral equation. The following lemma derives a concise formula for properly averaged Haar shift operators:

Lemma 3.1. Suppose the functions $h$ and $g$ are defined by (2.1),(2.2) and suppose $\gamma \in L_{\infty}\left(R_{+}\right)$. Then for any $x \neq y$, we have

$$
\int_{\{0,1\}^{\mathbb{Z}}} \int_{1}^{2} \sum_{I \in \mathbb{D}_{r, \beta}} \gamma(|I|) h_{I}(x) g_{I}(y) \frac{d r}{r} d \mathbb{P}(\beta)=\int_{0}^{\infty} \frac{\gamma(r)}{r^{2}}\left(h * g_{1}\right)\left(\frac{x-y}{r}\right) d r
$$

where $g_{1}(x) \equiv g(-x)$, and the functions $h_{I}, g_{I}$ are defined by (2.3). Here the series on the left of (3.2) is a.e. absolutely convergent.

Remark 3.3. This lemma appears in [2] for the case $\gamma \equiv 1$.

Remark 3.4. The notation $g_{1}$ is introduced in the right-hand side of (3.2) to emphasize the role of convolution.

Proof. The following calculation justifies the a.e. convergence of series:

$$
\begin{gathered}
\sum_{I \in \mathbb{D}_{r, \beta}}\left|\gamma(|I|) h_{I}(x) g_{I}(y)\right| \leq\|\gamma\|_{\infty} \sum_{\substack{I \in \mathbb{D}_{r, \beta} \\
x \in I, y \in I}}\left|h_{I}(x) g_{I}(y)\right| \\
\leq\|\gamma\|_{\infty}\|h\|_{\infty}\|g\|_{\infty} \sum_{\substack{I \in \mathbb{D}_{r, \beta} \\
x \in I,|I| \geq|x-y|}} \frac{1}{|I|} \leq \frac{2\|\gamma\|_{\infty}\|h\|_{\infty}\|g\|_{\infty}}{|x-y|} .
\end{gathered}
$$


Now recalling the definition of the dyadic grid $\mathbb{D}_{r, \beta}$ we get

$$
\begin{aligned}
\int_{\{0,1\}^{\mathbb{Z}}} \int_{1}^{2} \sum_{I \in \mathbb{D}_{r, \beta}} \gamma(|I|) h_{I}(x) g_{I}(y) \frac{d r}{r} d \mathbb{P}(\beta) \\
=\int_{\{0,1\}^{\mathbb{Z}}} \int_{1}^{2} \sum_{k \in \mathbb{Z}} \sum_{n \in \mathbb{Z}} \frac{\gamma\left(r 2^{n}\right)}{r 2^{n}} h\left(\frac{x}{r 2^{n}}-k-\sum_{i<n} 2^{i-n} \beta_{i}\right) \\
\quad \times g\left(\frac{y}{r 2^{n}}-k-\sum_{i<n} 2^{i-n} \beta_{i}\right) \frac{d r}{r} d \mathbb{P}(\beta) \\
=\int_{1}^{2} \sum_{n \in \mathbb{Z}} \frac{\gamma\left(r 2^{n}\right)}{r 2^{n}} \int_{\mathbb{R}} h\left(\frac{x}{r 2^{n}}-s\right) g\left(\frac{y}{r 2^{n}}-s\right) d s \frac{d r}{r} \\
=\sum_{n \in \mathbb{Z}} \int_{1}^{2} \frac{\gamma\left(r 2^{n}\right)}{r 2^{n}}\left(h * g_{1}\right)\left(\frac{x-y}{r 2^{n}}\right) \frac{d r}{r} \\
=\sum_{n \in \mathbb{Z}} \int_{2^{n}}^{2^{n+1}} \frac{\gamma(r)}{r^{2}}\left(h * g_{1}\right)\left(\frac{x-y}{r}\right) d r \\
=\int_{0}^{\infty} \frac{\gamma(r)}{r^{2}}\left(h * g_{1}\right)\left(\frac{x-y}{r}\right) d r .
\end{aligned}
$$

Having this lemma at hand, the claim of Theorem 2.4 is equivalent to the following: For $h$ and $g$ defined by (2.1) and (2.2), find a function $\gamma \in L_{\infty}(\mathbb{R})$ which would satisfy the following integral equation:

$$
K(x)=\int_{0}^{\infty} \frac{\gamma(r)}{r^{2}}\left(h * g_{1}\right)\left(\frac{x}{r}\right) d r
$$

for all $x>0$. The case $x<0$ would be satisfied automatically as both $K$ and $h * g_{1}$ are odd.

Step 2: Derivation of recursive equation. In this step we will use the functional equation (3.5) to get a recursive equation (3.7) for the coefficient function $\gamma$.

Differentiating (3.5) twice, we get

$$
K^{\prime \prime}(x)=\int_{0}^{\infty} \frac{\gamma(r)}{r^{4}}\left(h * g_{1}\right)^{\prime \prime}\left(\frac{x}{r}\right) d r, \quad x>0,
$$

or equivalently

$$
x^{3} K^{\prime \prime}(x)=\int_{0}^{\infty} t^{2} \gamma\left(\frac{x}{t}\right)\left(h * g_{1}\right)^{\prime \prime}(t) d t, \quad x>0 .
$$

Using the definitions (2.1), (2.2) for $h$ and $g$, we see that $h * g_{1}$ is a continuous, piecewise linear, odd function. The graph of $h * g_{1}$ on the positive axis is illustrated in Figure 1.

Thus, the function $\left(h * g_{1}\right)^{\prime \prime}$ is a linear combination of Dirac measures, which one can calculate from the graph above; in particular

$$
\left(h * g_{1}\right)^{\prime \prime}(x)=2 \delta(x-1 / 4)+18 \delta(x-1 / 2)-22 \delta(x-3 / 4)+7 \delta(x-1), \quad x>0,
$$

where $\delta$ is the usual Dirac delta function centered at the point 0 . 


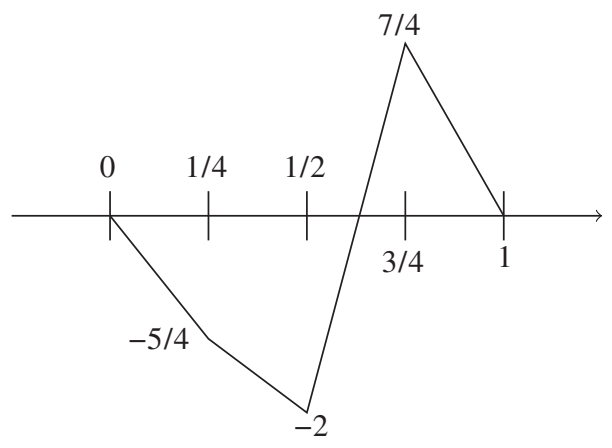

FIGURE $1 . h * g_{1}$ on the positive axis.

With this, (3.6) becomes

$x^{3} K^{\prime \prime}(x)=2\left(\frac{1}{4}\right)^{2} \gamma(4 x)+18\left(\frac{1}{2}\right)^{2} \gamma(2 x)-22\left(\frac{3}{4}\right)^{2} \gamma\left(\frac{4}{3} x\right)+7 \gamma(x), \quad x>0$.

Let's modify (3.7) to a form that will be more convenient to us. Denote

$$
m(x)=e^{3 x} K^{\prime \prime}\left(e^{x}\right)
$$

and

$$
c(x)=\gamma\left(e^{x}\right) .
$$

In terms of this new notation equation (3.7) becomes

$m(x)=\frac{1}{8} c(x+\ln 4)+\frac{9}{2} c(x+\ln 2)-22\left(\frac{3}{4}\right)^{2} c\left(x+\ln \left(\frac{4}{3}\right)\right)+7 c(x), \quad-\infty<x<\infty$.

Condition (2.6) of Theorem 2.4 provides that $m \in L_{\infty}(\mathbb{R})$. We want to find $c \in$ $L_{\infty}(\mathbb{R})$, which would solve (3.9).

Remark 3.10. In the case of the Hilbert transform we have $m(x) \equiv 2$; thus a constant function $c(x) \equiv C$ for a proper constant $C$ would solve (3.9).

Step 3: Fourier transform. We will use the Fourier transform in order to solve the recursive functional equation (3.9) . (Here we will deal with the Fourier transform of $L_{\infty}$ functions, which is understood in a distributional sense.)

Apply the Fourier transform to both sides of (3.9) to get

$$
m^{*}(\omega)=a(\omega) c^{*}(\omega)
$$

where

$$
a(\omega)=\frac{1}{8} e^{i \omega \ln 4}+\frac{9}{2} e^{i \omega \ln 2}-22\left(\frac{3}{4}\right)^{2} e^{i \omega \ln \left(\frac{4}{3}\right)}+7 .
$$

Now the function $a$ is a Fourier transform of a finite Borel measure on $\mathbb{R}$. Also note the following important property of $a$ : our choice of functions $h$ and $g$ provided that one of the terms of $a$ dominates the rest,

$$
12 \frac{3}{8}=22\left(\frac{3}{4}\right)^{2}>\frac{1}{8}+\frac{9}{2}+7=11 \frac{5}{8} .
$$

In particular, we have $|a(\omega)| \geq \frac{3}{4}$ for all $\omega$. 
Recall that the space of Fourier transforms of finite Borel measures on $\mathbb{R}$, equipped with the $L_{\infty}$-norms of these Fourier transforms, is a Banach algebra under pointwise multiplication. Therefore $a$ is invertible, too. (The inverse of $a$ can be written in terms of a Neumann series of exponents.) But this means that $a^{-1}$ is a multiplier of the space $L_{\infty}(\mathbb{R})$. Hence, there exists a function $c \in L_{\infty}(\mathbb{R})$, which solves the equation (3.11) and $\|c\|_{\infty}<C\|m\|_{\infty}$ (for some absolute constant $C)$. Using (3.8) we can further restore the coefficient function $\gamma$. It would solve the integral equation (3.6) and would satisfy the same bound as the function $c$, i.e.

$$
\|\gamma\|_{\infty} \leq C\|m\|_{\infty} .
$$

This fact, along with conditions (2.5) on the kernel $K$, is sufficient to make the integral in equation (3.5) convergent and to justify the passage from (3.6) back to (3.5).

Remark 3.13. The conditions (2.5) and (2.6) are somewhat necessary. Indeed, if some functions $h, g:[0,1] \rightarrow \mathbb{R}$ are constant on all dyadic intervals with sufficiently small length and if the coefficient function $\gamma$ is in $L_{\infty}(\mathbb{R})$, then Lemma 3.1 still holds. Thus, whatever kernel $K$ is restored by the averaging of the corresponding Haar shift operator, it must satisfy (3.5) and (3.6). If additionally $h$ is odd and $g$ is even with respect to the point $1 / 2$, then $h * g$ would be a piecewise linear function with bounded support, vanishing at 0 . So, (3.5) and (3.6) would imply that $K$ has to satisfy (2.5) and (2.6).

\section{ACKNOWLEDGMENT}

The author would like to thank Centre de Recerca Matemática at the Universitat Autónoma Barcelona for the invitation to participate in the research programme "Harmonic Analysis, Geometric Measure Theory and Quasiconformal Mappings".

\section{REFERENCES}

[1] Tadeusz Figiel, Singular integral operators: a martingale approach, Geometry of Banach spaces (Strobl, 1989), 1990, pp. 95-110. MR1110189 (94e:42015)

[2] Tuomas Hytönen, On Petermichl's dyadic shift and the Hilbert transform, C. R. Math. Acad. Sci. Paris 346 (2008), no. 21-22, 1133-1136 (English, with English and French summaries). MR2464252

[3] Oliver Dragičević and Alexander Volberg, Sharp estimate of the Ahlfors-Beurling operator via averaging martingale transforms, Michigan Math. J. 51 (2003), no. 2, 415-435. MR.1992955 (2004c:42030)

[4] Michael Lacey, Stephanie Petermichl, and Maria Carmen Reguera, Sharp $A_{2}$ Inequality for Haar Shift Operators, Math. Ann., to appear (2010), available at http://arxiv.org/abs/ 0906.1941

[5] Michael T. Lacey, Stefanie Petermichl, Jill C. Pipher, and Brett D. Wick, Multiparameter Riesz commutators, Amer. J. Math. 131 (2009), no. 3, 731-769. MR.2530853

[6] Michael T. Lacey, Jill C. Pipher, Stefanie Petermichl, and Brett D. Wick, Iterated Riesz Commutators: A Simple Proof of Boundedness, Proceedings of 8th Intl. Conf. on Harm. Analysis and PDE at El Escorial, Madrid (Spain), 2008, available at http://www.arxiv.org/ abs $/ 0808.0832$

[7] Andrei K. Lerner, Sheldy Ombrosi, and Carlos Pérez, $A_{1}$ bounds for Calderón-Zygmund operators related to a problem of Muckenhoupt and Wheeden, Math. Res. Lett. 16 (2009), no. 1, 149-156. MR2480568 (2010a:42052)

[8] F. Nazarov, S. Treil, and A. Volberg, The Bellman functions and two-weight inequalities for Haar multipliers, J. Amer. Math. Soc. 12 (1999), no. 4, 909-928. MR1685781(2000k:42009)

[9] _ Two weight inequalities for individual Haar multipliers and other well localized operators, Math. Res. Lett. 15 (2008), no. 3, 583-597. MR2407233(2009e:42031) 
[10] - The Tb-theorem on non-homogeneous spaces, Acta Math. 190 (2003), no. 2, 151-239. MR.1998349 (2005d:30053)

[11] Stefanie Petermichl, Dyadic shifts and a logarithmic estimate for Hankel operators with matrix symbol, C. R. Acad. Sci. Paris Sér. I Math. 330 (2000), no. 6, 455-460 (English, with English and French summaries). MR 1756958(2000m:42016)

[12] _ The sharp weighted bound for the Riesz transforms, Proc. Amer. Math. Soc. 136 (2008), no. 4, 1237-1249. MR2367098 (2009c:42034)

[13] S. Petermichl, S. Treil, and A. Volberg, Why the Riesz transforms are averages of the dyadic shifts?, Proceedings of the 6th International Conference on Harmonic Analysis and Partial Differential Equations (El Escorial, 2000) Publ. Mat., 2002, Vol. Extra, pp. 209-228. MR.1964822 (2003m:42028)

[14] S. Petermichl, The sharp bound for the Hilbert transform on weighted Lebesgue spaces in terms of the classical $A_{p}$ characteristic, Amer. J. Math. 129 (2007), no. 5, 1355-1375. MR2354322 (2008k:42066)

School of Mathematics, Georgia Institute of Technology, Atranta, Georgia 30332

E-mail address: armenv@math.gatech.edu

E-mail address: armen@math.brown.edu 